\title{
Infecção tripla por Trypanosoma cruzi, Plasmodium vivax e P. falciparum: relato de caso
}

\author{
Triple infection by Trypanosoma cruzi, Plasmodium vivax and P. falciparum: case report
}

Andrea Silvestre Lobão Costa ${ }^{1}$; Valdira Cardoso Santos²; Paoola Cristina Vieira Amorim; José Eduardo Santos ${ }^{4}$; Ana Yecê das Neves Pinto

unitermos
Doença de Chagas
Malária
Trypanosoma cruzi
Rhodnius
Coinfecção

\section{resumo}

O presente registro acerca da identificação de infecção aguda de dois plasmódios e um Trypanosoma constitui evento raro. Pré-escolar, sexo feminino, 5 anos de idade, apresentou síndrome febril; foi submetida a exame de gota espessa no qual foram identificadas formas assexuadas e sexuadas de Plasmodium vivax e $P$. falciparum, respectivamente, além de tripomastigotas sanguíneos de Trypanosoma cruzi. No peridomicílio, foram encontrados insetos da espécie Rhodnius sp. Os autores reforçam a importância dos estudos dos ciclos peridomiciliares de T. cruzi em ambientes silvestres na Amazônia e discutem a importância da estratégia de vigilância continuada de Trypanosomas spp. nos exames de gota espessa.

\section{abstract}

This report describes a rare case of acute infection caused by two Plasmodia and one Trypanosoma. 5 year-old female patient attending kindergarten presented persistent fever syndrome. She was submitted to thick smear exam, in which asexual and sexual forms of Plasmodium vivax and $\mathrm{P}$. falciparum were detected, respectively, as well as trypomastigotes of Trypanosoma cruzi. Rhodnius sp. triatomines were found in the vicinity. The authors reinforce the importance of investigating the domiciliary cycles of T. cruzi in the Amazon region. Moreover, we discuss the importance of continuous monitoring of Trypanosomas spp. in thick smear exams. key words

Chagas disease

\section{Malaria}

Trypanosoma cruzi

Rhodnius

Coinfection

\footnotetext{
1. Especialista em Doenças Tropicais pela Universidade Federal do Pará (UFPA); biomédica do Laboratório Central do Estado do Pará.

2. Mestre em Epidemiologia dos Agentes Infecciosos e Parasitários pela UFPA; farmacêutica da Fundação Hospital de Clínicas Caspar Vianna.

3. Mestre em Zoologia pela UFPA; coordenadora estadual da Entomologia no Estado do Pará.

4. Agente de Endemias da Secretaria Municipal de Saúde de Anajás.

5. Doutora em Medicina pela Fundação Oswaldo Cruz; pesquisadora em Saúde Pública do Instituto Evandro Chagas.
} 


\section{Introdução}

O incremento das estatísticas de casos de doença de Chagas (DC) com ênfase em fase aguda, a partir de 1992, na Amazônia, determinou inúmeras iniciativas de controle idealizadas para a região, sendo a mais importante delas a estratégia para o diagnóstico precoce. Assim, a busca de $T$. cruzi em lâminas de gota espessa foi uma importante medida idealizada desde 2002, mas foi colocada em prática apenas em $2007^{(4)}$. Nessa estratégia, a partir de treinamentos-piloto realizados nos seis estados da região amazônica, foram habilitados 238 microscopistas de malária para identificação de $T$. cruzi em lâminas de gota espessa, de 2008 a 2010, desencadeados pelo Laboratório de Doenças Parasitárias da Fundação Oswaldo Cruz (FIOCRUZ); após, o Laboratório Central do Estado do Pará (LACEN-PA) deu continuidade aos procedimentos. A despeito de a estratégia ter sido implantada somente em 2008, já havia referências na Amazônia brasileira sobre o encontro casual em gota espessa da infecção concomitante de $T$. cruzi e plasmódio em relatos anteriores ${ }^{(11)}$.

A cidade de Anajás, na llha do Marajó, considerada prioritária para controle de malária, atingiu em 2009 um índice parasitário anual (IPA) extremo de 951 casos em 1.000 habitantes ${ }^{(16)}$. Por outro lado, a frequência de casos de DC aguda (DCA) é baixa, tendo sido notificados 10 casos entre 2006 e 2010.

O presente relato raríssimo acerca da identificação de tripla infecção por dois plasmódios e um Trypanosoma em uma criança tem por objetivo destacar a importância da necessidade de capacitação sustentada de microscopistas para a identificação de Trypanosoma em pacientes febris com suspeita de malária, além de levantar discussões sobre essa casual concomitância de infecções por hemoparasitas desde que a estratégia foi instituída.

\section{Relato do caso}

Pré-escolar, 5 anos de idade, sexo feminino, natural e residente na comunidade de Caetanópolis, rio Mocoões, Anajás-PA. Apresentava febre persistente, astenia, cefaleia, anorexia, tonturas, náuseas, diarreia e discreta tosse seca durante oito dias. Não foi registrado nenhum sinal de porta de entrada no período. No dia 16 de julho de 2010, procurou o setor de endemias do município para tentativa diagnóstica de malária por gota espessa. Nesta, foram identificados trofozoítos de $P$. vivax, gametócitos de $P$. falciparum e tripomastigotas de $T$. cruzi. A lâmina foi enviada ao LACEN-PA para revisão, confirmando o diagnóstico (Figuras 1 e 2). No mesmo dia, a menor recebeu tratamento com coartem, primaquina e benzonidazol; realizou sorologia para pesquisa de anticorpos anti$T$. cruzi, utilizando-se as técnicas de imunofluorescência indireta (IFI), hemaglutinação indireta (HI) e ensaio imunoenzimático ligado à enzima (ELISA), além de exames eletrocardiográficos e ecocardiográficos em Belém.

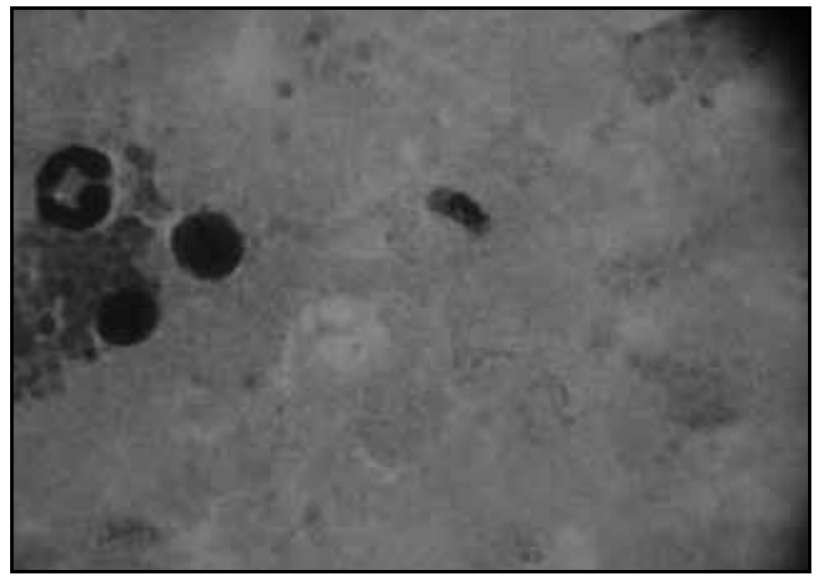

Figura 1 - Gametócito de Plasmodium falciparum observado em lâmina corada. MO, 100x

MO: microscopia óptica.

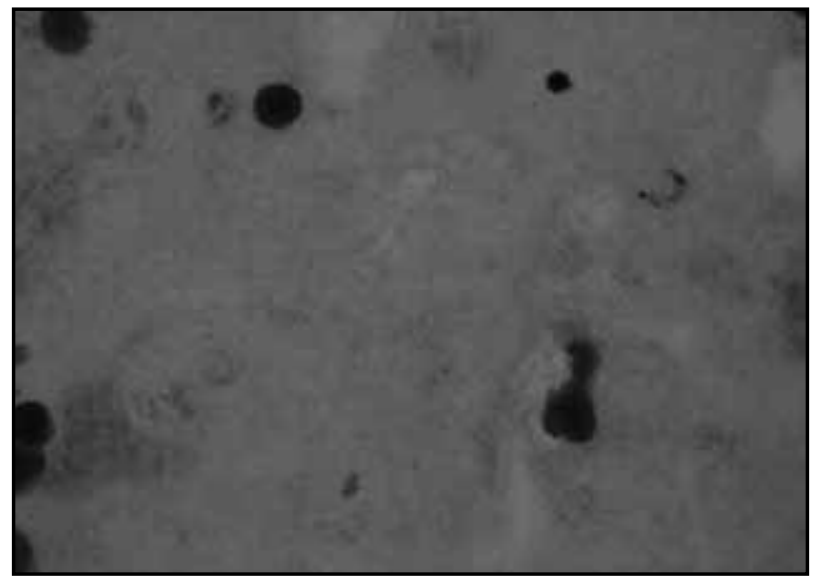

Figura 2 - Trofozoíta de Plasmodium vivax e tripomastigota de Trypanosoma cruzi em lâmina corada. M0, 100x

MO: microscopia óptica.

Segundo sua genitora, a menor cumpriu o tratamento antimalárico corretamente por sete dias. Entretanto, o tratamento com benzonidazol foi suspenso no hospital de Belém por motivos desconhecidos, tendo a criança feito apenas seis dias dessa medicação. Os exames complementares do período demonstraram anemia, discreta leucocitose e exames cardiológicos normais (Tabela). A menor tornou-se afebril e retornou ao município de origem. 


\section{Resultados diagnósticos, complementares e de acompanhamento antes e após tratamentos com} Tabela benzonidazol e coartem

\begin{tabular}{|c|c|c|c|}
\hline $\begin{array}{l}\text { Exames diagnósticos, radiológicos } \\
\text { e clinicocardiológicos }\end{array}$ & $\begin{array}{l}\text { Resultados de } \\
\text { diagnóstico }\end{array}$ & Reavaliação* & $\begin{array}{c}\text { Resultados após } \\
\text { duplo tratamento** }\end{array}$ \\
\hline Gota espessa & T. cruzi + PV + Fg & $40 F+5 F g$ & Negativo \\
\hline Concentrado leucocitário & Prejudicado & Negativo & NR \\
\hline IFI IgM & 160 & Negativo & Negativo \\
\hline$|F| \lg G$ & 160 & 80 & Negativo \\
\hline Hemaglutinação indireta & Não reagente & Não reagente & Negativo \\
\hline ELISA & Não reagente & Não reagente & NR \\
\hline Hemoglobina (\%) & 10,8 & 9,5 & 11,6 \\
\hline Leucócitos $\left(\mathrm{mm}^{3}\right)$ & 10.200 & 8.200 & 9.350 \\
\hline Eosinófilos $\left(\mathrm{mm}^{3}\right)$ & 510 & 902 & 561 \\
\hline Segmentados $\left(\mathrm{mm}^{3}\right)$ & 5.854 & 3.444 & 6.545 \\
\hline Linfócitos (mm³) & 3.162 & 3.362 & 1.870 \\
\hline Monócitos $\left(\mathrm{mm}^{3}\right)$ & 591 & 492 & 374 \\
\hline Plaquetas $\left(\mathrm{mm}^{3}\right)$ & 252.000 & 324.000 & 301.000 \\
\hline Alaninoaminotransferases (UVI) & 11 & 16 & 29 \\
\hline Aspartatoaminotransferases (UVI) & 20 & 29 & 24 \\
\hline Rx de tórax & Normal & Normal & NR \\
\hline Eletrocardiograma de repouso & Sem alterações & Sem alterações & Sem alterações \\
\hline Ecocardiograma & Normal & Normal & Normal \\
\hline
\end{tabular}

Cerca de 60 dias após o diagnóstico, foi reenviada pelo setor de endemias municipal para consulta no Instituto Evandro Chagas (IEC), em virtude da persistência de anemia, anorexia, astenia e calafrios, para avaliação da necessidade de reintrodução da medicação antichagásica. Foi submetida à nova avaliação e apresentou no exame parasitológico trofozoítas de $P$. falciparum e ausência de $T$. cruzi. O exame sorológico demonstrou persistência da resposta sorológica anômala anterior (IFI positivo e HI e ELISA negativos) para imunoglobulina da classe $\mathrm{G}(\mathrm{lgG})$ anti-T. cruzi (Tabela).

No mesmo período (23 de setembro de 2010), o tratamento antimalárico foi refeito e o antichagásico, reiniciado, conforme apresentado na Tabela. Foram utilizados coartem e benzonidazol durante três e 60 dias, respectivamente. Realizou-se o seguimento sob o protocolo clínico de pesquisa no IEC, incluindo coleta de sangue periférico para exame de gota espessa semanalmente por cinco semanas, após final do tratamento antimalárico, com todos os resultados negativos. Também foram realizadas sorologias seriadas para anticorpos lgG anti-T. cruzi. Apenas o resultado da avaliação mais recente referente a esse seguimento está demonstrado na Tabela.

A investigação epidemiológica identificou moradia construída em madeira, coberta por telhas, sem qualquer tipo de vedação entre tábuas e telhado, em região ribeirinha. Foi identificado no peridomicílio, triatomíneo silvestre do gênero Rhodnius, o qual não foi examinado por estar morto e prejudicado para exame. Nenhum dos outros sete moradores da residência da criança apresentou os sintomas. Todos foram submetidos a exames sorológicos pela vigilância epidemiológica municipal e apresentaram resultados negativos.

\section{Discussão}

Em Anajás, mudanças ambientais e climáticas resultantes da degradação ambiental, somadas à adaptação de vetores 
aos ambientes modificados pelo homem, condições precárias de habitação e trabalho, acesso difícil, precariedade dos serviços de saúde e urbanização descontrolada, possibilitam a perpetuação de níveis elevados de endemicidade de malária, o principal problema de saúde pública da cidade $^{(1,13)}$. Contrariamente, a incidência da DC é baixa, apesar de esses índices estarem aumentando na Amazônia com o surgimento de casos em fase aguda, sejam isoladamente ou sob a forma de $\operatorname{surtos}^{(20)}$. Desde os primeiros relatos até o ano de 2010, 1.125 casos de DC foram registrados, com especial referência a transmissão por via oral ${ }^{(6)}$.

Em áreas endêmicas para malária, é relativamente comum a infecção mista por $P$. falciparum e $P$. vivax ${ }^{(5)}$. Técnicas de biologia molecular apontam para o subdiagnóstico de infecções mistas pela microscopia, mostrando que as frequências das infecções mistas podem ser superiores àquelas registradas $^{(2,8,17)}$. Por outro lado, a infecção simultânea por dois hemoparasitas diferentes constitui um evento teoricamente raro, dada a necessidade de exposição do hospedeiro a dois vetores diferentes e infectados quase simultaneamente, mesmo diante da possibilidade de transmissão oral para DCA, na qual há necessidade indireta do vetor ou reservatório. Assim, o presente relato soma-se a dois registros anteriores de coinfecção por malária e DC ocorridos em área urbana e periurbana ${ }^{(11)}$.

O diagnóstico de $T$. cruzi foi confirmado devido à presença de formas tripomastigotas e apresentou cinetoplasto terminal de aspecto grosseiro, demonstrando, indubitavelmente, por sua morfologia, não se tratar de $T$. rangeli(3).

Quanto ao diagnóstico sorológico nas descrições de áreas endêmicas clássicas, a maioria dos pacientes com DCA desenvolve anticorpos lgG anti-T. cruzi e mantém títulos de anticorpos após o tratamento, em períodos variáveis entre 3 e 5 anos de idade, sendo essa resposta utilizada como critério de cura em orientações técnicas ${ }^{(15)}$. Na Amazônia, pacientes tratados em fase aguda com acompanhamento demonstraram similaridade aos registros de área endêmica, tendo sido descritos níveis detectáveis de anticorpos $\lg \mathrm{C}$ anti-T. cruzi persistentes por um período mínimo de quatro $\operatorname{anos}^{(10)}$. Outra experiência que demonstra negativação de anticorpos sete anos após o tratamento em pacientes avaliados por soroprevalência pontual, ou seja, sem detecção do momento da negativação(20).

No presente relato, a criança desenvolveu resposta sorológica incomum, mantendo apenas uma das provas sorológicas positivas para os anticorpos IgG anti-T. cruzi (IFI positiva e HI e ELISA negativos) até sua total negativação, em um período curto de nove meses. Essa resposta é considerada discordante e poderia ser explicada pela presença de três parasitas simultâneos. Descarta-se, assim, a cura sorológica obtida em pouco tempo com tratamento específico, contrariamente ao que se tem descrito, tanto pra Amazônia ${ }^{(10)}$ quanto para áreas endêmicas. Já foi descrita para as infecções mistas de plasmódios a relativa competição entre eles quando coexistem agudamente, com reflexões clínicas dessa competição( ${ }^{(2,9)}$ e, possivelmente, reflexos na resposta imune ${ }^{(18)}$.

Para avaliação complementar, procedemos com exames inespecíficos. Entre os achados hematológicos mais comumente encontrados em pacientes com malária, estão a anemia e a trombocitopenia ${ }^{(7)}$. Da mesma forma, na DCA, também se observa anemia como um indicativo clínico causador de viés na suspeita diagnóstica com malária ${ }^{(12)}$. No presente relato, a anemia foi identificada na criança. O restabelecimento aos níveis próximos do normal, após a conclusão de ambos os esquemas terapêuticos para malária e DC, foi mais bem observado somente depois do tratamento antichagásico (Tabela). A principal espécie anofelina responsável pela transmissão de malária em Anajás é o Anopheles Darlingi(14), com altas taxas de paridade e de infectividade. Para a DCA, no caso estudado, a transmissão foi indefinida, enfatizando-se o encontro do triatomínio observado no peridomicílio da criança e a não ocorrência de surto(19).

A pesquisa de $T$. cruzi em gota espessa, mesmo tendo sensibilidade inferior aos métodos de concentração, vem sendo amplamente utilizada na Amazônia devido a sua praticidade e disponibilidade da infraestrutura de laboratórios de microscopia já implantados ${ }^{(4)}$. Um desafio a ser considerado é a alta rotatividade de microscopistas, exigindo capacitações e supervisões contínuas a fim de garantir a qualidade diagnóstica, aumentando a sensibilidade de identificação das coinfecções.

\section{Agradecimentos}

Ao Setor de Endemias da Secretaria Municipal de Saúde de Anajás; ao Laboratório de Revisão do Município de Anajás; ao Serviço de Parasitologia e Divisão de Entomologia do LACEN/PA; aos Laboratórios de Ensaios Clínicos em Malária e de Doença de Chagas do IEC; à Gerência Técnica de Doença de Chagas da Secretaria Estadual de Saúde do Pará (SESPA) pelo fornecimento de dados numéricos de casos; à Fundação Hospital de Clínicas Gaspar Viana, em Belém, pelos exames cardiológicos; e ao Antonio Carlos da Silveira (in memoriam). 


\section{Referências}

1. CASTRO, M. C.; SINGER, B. H. Meio ambiente e saúde: metodologia para análise espacial da ocorrência de malária em projetos de assentamento. R Bras Est Pop, São Paulo, v. 24, p. 247-62, 2007.

2. COSTA, M. R. F.; VIEIRA, P. P. R.; FERREIRA, C. O.; LACERDA, M. V. G.; ALECRIM, W. D.; ALECRIN, M. G. C. Diagnóstico molecular da malária em uma unidade de atenção terciária na Amazônia brasileira. Revista da Sociedade Brasileira de Medicina Tropical, v. 41, p. 381-5, 2008.

3. COURA, J. R.; FERNANDES, O.; ARBOLEDA, M.; BARRET, T.; CARRARA, N.; DEGRAVE, W. Human infection by Trypanosoma rangeli in the Brazilian Amazon. Transactions of the Royal Society of Tropical Medicine and Hygiene, v. 90, p. 278-9, 1996.

4. De SOUZA, D. O.; De OLIVEIRA, S. M.; VIVALDINI, S.; SILVEIRA, A. C.; JUNQUEIRA, A.; VIÑAS, P. A. Diagnóstico parasitológico direto de casos agudos de infecção por Trypanosoma cruzi em lâmina de malária na Amazônia brasileira: avaliação do treinamento e do potencial do método. Revista da Sociedade Brasileira de Medicina Tropical, v. 42, p. 425, 2009.

5. FERREIRA, M. S. Malária: patologia, fisiopatologia, quadro clínico e diagnóstico. In: Veronesi: tratado de infectologia. 4. ed. São Paulo: Atheneu, 2010, v. 2.

6. FRAIHA NETO, H.; PINTO, A. Y. N.; VALENTE, V. C.; VALENTE, S. A. S.; LEÃO, R. N. Q. Doença de Chagas. In: LEÃO, R. N. Q. (Ed.). Doenças infecciosas e parasitárias - enfoque amazônico. Belém: CEJUP, 2011.

7. LACERDA, M. V. G. Manifestações clínicas e patológicas da plaquetopenia na malária. 2007. Tese de Doutorado Universidade de Brasília, Brasília. 2007.

8. MAY, J. et al. High rate of mixed and subpatent malarial infections in southwest Nigeria. The American Society of Tropical Medicine and Hygiene, v. 61, p. 339-43, 1999.

9. MCQUEEN, G. F.; MCKENZIE, F. E. Competition for red blood cells can enhance Plasmodium vivax parasitemia in mixed-species malaria infections. Am J Trop Med Hyg, v. 75, p. 112-25, 2006.

10. PINTO, A. Y. N.; FERREIRA JR, A. G.; VALENTE, V. C.; HARADA, G. S.; VALENTE, S. A. S. Urban outbreak of acute Chagas disease in Amazon region of Brazil: four-year follow-up after treatment with benznidazole. Revista Panamericana de Salud Pública, v. 25, n. 1, p. 77-83, 2009.

11. PINTO, A. Y. N. et al. Infecção aguda por T. cruzie P. vivax - relato de dois casos. Revista da Sociedade Brasileira de Medicina Tropical, v. 40, p. 36, 2007.
12. PINTO, A. Y. N.; VALENTE, S. A. S.; VALENTE, V. C.; FERREIRA JR, A. G.; COURA, J. R. Fase aguda da doença de Chagas na Amazônia brasileira. Estudo de 233 casos do Pará, Amapá e Maranhão observados entre 1988 e 2005. Revista da Sociedade Brasileira de Medicina Tropical, v. 41, p. 602-14, 2008.

13. ROSA, C. C.; EDNA, R. C.; ROSA, A. C. Saúde, trabalho e meio ambiente: políticas públicas na Amazônia. Belém: Universidade Federal do Pará. Núcleo de Altos Estudos Amazônicos, p. 123, 2002.

14. SANTOS, R. L. C.; SUCUPIRA, I. M. C.; LACERDA, R. N. L.; FAYAL, A. S.; PÓVOA, M. M. Inquérito entomológico e infectividade durante epidemia de malária no município de Anajás, Estado do Pará. Revista da Sociedade Brasileira de Medicina Tropical, v. 38, p. 202-4, 2005.

15. SECRETARIA DE VIGILÂNCIA EM SAÚDE. Ministério da Saúde. Brasil. Consenso Brasileiro em Doença de Chagas. Revista da Sociedade Brasileira de Medicina Tropical, v. 38, p. 30, 2005.

16. SIVEP-MALÁRIA. Disponível em: http://www.webcitation. org/getfile?fileid=37cb614143090770979ba9a8d33 25a07e3fd5204. Acesso em: 13 de junho de 2011.

17. STEENKESTE, N. et al. Sub-microscopic malaria cases and mixed malaria infection in a remote area of high malaria endemicity in Rattanakiri province, Cambodia: implication for malaria elimination. Malaria Journal, v. 9, p. 108, 2010.

18. TAYLOR, H. L.; WALLIKER, D.; READ, A. Mixed-genotype infections of malaria parasites: within-host dynamics and transmission success of competing clones. Proceedings Biological Sciences, v. 264, p. 22, 1996.

19. VALENTE, S. A. S.; VALENTE, V. C.; PINTO, A. Y. N. Epidemiologia e transmissão oral da doença de Chagas na Amazônia brasileira. In: Organización Panamericana de la Salud. Unidad Regional de Prevention y Control de Enfermedades Transmisibles (DPC/CD/CHA), Grupo Técnico Especializado en inocuidad de Alimentos. Informe de la consulta técnica en epidemiologia, prevención y manejo de la transmisión de la enfermedad de Chagas como enfermedad transmitida por alimentos (ETA). Rio de Janeiro, 2006; p. 46.

20. VALENTE, S. A. S. et al. Analysis of an acute Chagas disease outbreak in the Brazilian Amazon: human cases, triatomines, reservoir mammals and parasites. Transactions of the Royal Society of Tropical Medicine and Hygiene, v. 103, p. 291-7, 2009. 